\title{
THE SYMMETRIC PLURICOMPLEX GREEN FUNCTION
}

\author{
URBAN CEGRELL \\ Department of Mathematics, University of Umea \\ S-90187 Umeå, Sweden
}

1. Introduction. Let $\Omega$ be an open and connected set in $\mathbb{R}^{n} ; x_{0} \in \Omega$. Then the classical Green function $G_{\Omega}\left(x, x_{0}\right)$ is the solution to the Dirichlet problem

$$
\left\{\begin{array}{l}
G_{\Omega}\left(x, x_{0}\right)=0, \quad \forall x \in \partial \Omega \\
\Delta_{x} G\left(x, x_{0}\right)=\delta_{x_{0}} .
\end{array}\right.
$$

In [7], Klimek introduced the pluricomplex Green function $g_{\Omega}$, that can be defined as solution to

$$
\left\{\begin{array}{l}
g_{\Omega}\left(z, z_{0}\right) \in P S H(\Omega) \\
g_{\Omega}\left(z, z_{0}\right)=0, \quad \forall z \in \partial \Omega \\
\left(d d_{z}^{c} g_{\Omega}\left(z, z_{0}\right)\right)^{n}=(2 \pi)^{n} \delta_{z_{0}}
\end{array}\right.
$$

where $\Omega$ is a domain (open, bounded, and connected set) in $\mathbb{C}^{n} ; z_{0} \in \Omega$.

An alternative definition of $g_{\Omega}$ for any domain $\Omega$ in $\mathbb{C}^{n}, z_{0} \in \Omega$ is

$$
\begin{aligned}
& g_{\Omega}\left(z, z_{0}\right)=\sup \left\{\varphi(z) ; \varphi \in \operatorname{PSH}(\Omega), \varphi \leq 0, \varphi(z)-\log \left|z-z_{0}\right|\right. \\
& \text { bounded above near } \left.z=z_{0}\right\} \text {. }
\end{aligned}
$$

It is well known that the classical Green function is symmetric: $G_{\Omega}\left(x, x_{0}\right)=$ $G_{\Omega}\left(x_{0}, x\right)$. However, the pluricomplex Green function need not be symmetric.

It was shown by Bedford and Demailly [2] that there exists a strictly pseudoconvex smooth $\Omega$ such that $g_{\Omega}\left(z, z_{0}\right) \neq g_{\Omega}\left(z_{0}, z\right)$.

2. The symmetric pluricomplex Green function. In [3], we introduced the symmetric pluricomplex Green function $W_{\Omega}(z, \omega)$,

$$
\begin{gathered}
W_{\Omega}(z, \omega)=\sup \{\varphi(z, \omega) \in 2-P S H(\Omega \times \Omega), \quad \varphi \leq 0, \\
\varphi(z, \omega) \leq \log |z-\omega|-\log \max [d(z, C \Omega), d(\omega, C \Omega)]\} .
\end{gathered}
$$

1991 Mathematics Subject Classification: Primary 32F05.

The paper is in final form and no version of it will be published elsewhere. 
Here, $2-P S H(\Omega \times \Omega)$ denotes the subharmonic functions that are also separately plurisubharmonic. The purpose of this note is to consider some basic properties of $W_{\Omega}$.

Definition. A domain $\Omega$ is said to be strongly hyperconvex if $W_{\Omega}(z, \omega)$ is an exhaustion function for $\Omega$ for each fixed $\omega \in \Omega$.

Rem a r k. Every strictly pseudoconvex set is strongly hyperconvex.

Lemma 1. Suppose $\varphi$ is plurisubharmonic near zero and that $|\varphi(z)-\log | z||<$ $K$ near zero for some constant $K$. Then $\mu(0) \geq(2 \pi)^{n}$ where $\mu$ is the weak ${ }^{*}$-limit of $\left(d d^{c} \max [\varphi, t]\right)^{n}, t \rightarrow-\infty$.

Proof. Let $1>r>0$ so that $\varphi$ is plurisubharmonic and so that

$$
|\varphi(z)-\log (z)| \leq K \quad \text { on } B(0, r) .
$$

Given $0<\epsilon<1$, then

$$
\begin{aligned}
|z|>e^{\frac{-1}{\epsilon}} \Rightarrow \log |z| \geq \frac{-1}{\epsilon} \Rightarrow-\epsilon \log |z|<1 \Rightarrow(\varphi-\log |z|>-K) \Rightarrow \\
\varphi-\log |z|>-(K+1)-\epsilon \log |z| \Rightarrow \varphi(z)>(1-\epsilon) \log |z|-(K+1) .
\end{aligned}
$$

Thus

$$
\Omega_{\epsilon}=\{z \in B(0, r) ; \varphi(z)<(1-\epsilon) \log |z|-(K+1)\}
$$

is a neighborhood of zero and relatively compact in $B(0, r)$ if $e^{-\frac{1}{\epsilon}}<r$.

Let $t<\inf _{z \in \partial \Omega_{\epsilon}}(1-\epsilon) \log |z|-(K+1)=\delta<0$ and define $\varphi_{2 t}=\max [\varphi, 2 t]$.

Then $\Omega_{\epsilon}^{t}=\left\{z \in B(0, r) ; \varphi_{2 t}<\max [(1-\epsilon) \log |z|-(K+1), t]\right\}$ is a neighborhood of zero and relatively compact in $B(0, r)$. Thus

$$
\int_{\Omega_{\epsilon}^{t}}\left(d d^{c} \varphi_{t}\right) \geq \int_{\Omega_{\epsilon}^{t}}\left(d d^{c} \max [(1-\epsilon) \log |z|-K+1, t]\right)^{n}=(2 \pi)^{n}(1-\epsilon)^{n}
$$

and since $\Omega_{\epsilon}^{t} \subset \Omega_{\epsilon} \subset B\left(0, e^{-\frac{1}{\epsilon}}\right)$,

$$
\int_{B\left(0, e^{-\frac{1}{\epsilon}}\right)}\left(d d^{c} \varphi_{2 t}\right)^{n} \geq(2 \pi)^{n}(1-\epsilon)^{n} .
$$

So if $\mu$ is the weak*-limit of $\left(d d^{c} \varphi_{2 t}\right)^{n}, t \rightarrow-\infty$, then $\mu(0) \geq(2 \pi)^{n}$ which proves the lemma.

TheOREM 1. Suppose $\Omega$ is strongly hyperconvex. Then $g_{\Omega} \geq W_{\Omega}$ with equality if and only if

$$
\tau(z)=\int_{\Omega}\left(d d_{\xi}^{c} \max \left[W_{\Omega}(z, \xi),-1\right]\right)^{n}=(2 \pi)^{n}, \quad \forall z \in \Omega .
$$

Proof (cf. [3, Prop. VII:2). Note first that $\int_{\Omega}\left(d d_{z}^{c} \max \left[g_{\Omega}(z, \xi), t\right]\right)^{n}=$ $(2 \pi)^{n}$, 
$\forall t<0, \forall z \in \Omega$ and that

$$
\tau(z)=\int_{\Omega}\left(d d_{\xi}^{c} \max \left[W_{\Omega}(z, \xi), t\right]\right)^{n}
$$

is independent of $t$ for all negative $t$. Also, it follows from definitions that $g_{\Omega} \geq$ $W_{\Omega}$. It follows from Lemma 1 that $\tau(z) \geq(2 \pi)^{n}$ with equality if $W_{\Omega} \equiv g_{\Omega}$.

On the other hand, assume $\tau(z) \equiv(2 \pi)^{n}$. Again, by Lemma $1,\left(d d^{c} W_{\Omega}(z, \xi)\right)^{n}$ $=0$ on $z \neq \xi$. Let $\xi \in \Omega$ be given and consider for $0<\epsilon<1,(1-\epsilon) W_{\Omega}(z, \xi)$. Then $(1-\epsilon) W_{\Omega}(z, \xi)=0$ on $\partial \Omega,(1-\epsilon) W_{\Omega}(z, \xi) \geq g_{\Omega}(z, \xi)$ for $z$ near $\xi$. Since $\left(d d_{z}^{c}(1-\epsilon) W_{\Omega}(z, \xi)\right)^{n}=0$ outside $\xi,(1-\epsilon) W_{\Omega}(z, \xi) \geq g_{\Omega}(z, \xi)$ on $\Omega$. Letting $\epsilon \searrow 0$, we find that $W_{\Omega}=g_{\Omega}$.

Lemma 2. Let $\Omega_{1} \subset \mathbb{C}^{n}, \Omega_{2} \subset \mathbb{C}^{n}$ be two open and connected sets. Then

Proof.

$$
\max \left(W_{\Omega_{1}}, W_{\Omega_{2}}\right) \leq W_{\Omega_{1} \times \Omega_{2}}
$$

$$
\begin{aligned}
0 & \geq \max \left[W_{\Omega_{1}}\left(z_{1}, \omega_{1}\right), W_{\Omega_{2}}\left(z_{2}, \omega_{2}\right)\right] \\
& \leq \max \left(\log \left|z_{1}-\omega_{1}\right|-\log \max \left[d\left(z_{1}, C \Omega_{1}\right), d\left(\omega_{1}, C \Omega_{1}\right)\right],\right. \\
& \left.\quad \log \left|z_{2}-\omega_{2}\right|-\log \max \left[d\left(z_{2}, C \Omega_{2}\right), d\left(\omega_{2}, C \Omega_{2}\right)\right]\right) \\
& \leq \log \left|\left(z_{1}, z_{2}\right)-\left(\omega_{1}, \omega_{2}\right)\right|-\log \min \left[d\left(z_{1}, C \Omega_{1}\right), d\left(\omega_{1}, C \Omega_{1}\right), d\left(z_{2}, C \Omega_{1}\right), d\left(\omega_{2}, C \Omega_{2}\right)\right]
\end{aligned}
$$

so the inequality now follows from the definition of $W_{\Omega_{1} \times \Omega_{2}}$ via [3, Cor. VII:1].

Example. Denote by $C_{\Omega}$ the Carathéodory pseudodistance on $\Omega$. We give an example of a bounded pseudoconvex set $\Omega$, such that

$$
\log \tanh C_{\Omega} \neq W_{\Omega} \neq g_{\Omega} .
$$

Let $\Omega_{1}=\left\{z \in \mathbb{C} ; \frac{1}{2}<|z|<1\right\}$ and let $\Omega_{2}$ be any strictly pseudoconvex domain where $W_{\Omega_{2}}\left(z_{2}^{0}, \omega_{2}^{0}\right)<g_{\Omega_{2}}\left(z_{2}^{0}, \omega_{2}^{0}\right)$ for a point $\left(z_{2}^{0}, \omega_{2}^{0}\right) \in \Omega_{2} \times \Omega_{2}$ (by [2], such a set exists). Note first that $W_{\Omega_{1}}=g_{\Omega_{1}}$ and that

$$
W_{\Omega_{1}}\left(z_{1}, \omega_{1}\right)>\log \tanh C_{\Omega}\left(z_{1}, w_{1}\right), \forall z_{1} \neq \omega_{1} \in \Omega_{1}
$$

(cf. Klimek [7], p. 234-235]).

Then $\log \tanh C_{\Omega_{1} \times \Omega_{2}}\left(\left(z_{1}, z_{2}\right),\left(\omega_{1}, z_{2}\right)\right)<W_{\Omega_{1}}\left(z_{1}, \omega_{1}\right) \leq \max \left[W_{\Omega_{1}}\left(z_{1}, w_{1}\right)\right.$, $\left.W_{\Omega_{2}}\left(z_{2}, z_{2}\right)\right] \leq W_{\Omega_{1} \times \Omega_{2}}\left(\left(z_{1}, z_{2}\right),\left(\omega_{1}, z_{2}\right)\right)$ by Lemma 2 . Thus $\log \tanh C_{\Omega_{1} \times \Omega_{2}} \neq$ $W_{\Omega_{1} \times \Omega_{2}}$; it remains to prove that $W_{\Omega_{1} \times \Omega_{2}} \neq g_{\Omega_{1} \times \Omega_{2}}$. Suppose $W_{\Omega_{1} \times \Omega_{2}} \equiv g_{\Omega_{1} \times \Omega_{2}}$. Since $\Omega_{1}$ and $\Omega_{2}$ are pseudoconvex, it follows from Theorem 9.6 in [6] that $g_{\Omega_{1} \times \Omega_{2}}=\max \left[g_{\Omega_{1}}, g_{\Omega_{2}}\right]$ so $W_{\Omega_{1} \times \Omega_{2}}\left(\left(z_{1}, z_{2}\right),\left(z_{1}, \omega_{2}\right)\right)=g_{\Omega_{2}}\left(z_{2}, \omega_{2}\right)$ is plurisubharmonic in $\omega_{2}$ which is a contradiction to the assumption

$$
W_{\Omega_{2}}\left(z_{2}^{0}, \omega_{2}^{0}\right)<g_{\Omega_{2}}\left(z_{2}^{0}, \omega_{2}^{0}\right)
$$

by Proposition VII:2 in [3]. 
3. Some estimates. If $\Omega$ is a domain in $\mathbb{R}^{n}$, regular for the classical Dirichlet problem, then for every function $\varphi$, subharmonic near $\bar{\Omega}$ we have the Riesz representation formula:

$$
\varphi(\omega)=\int_{\Omega} G(\xi, \omega) \Delta \varphi(\xi)+\int_{\partial \Omega} \varphi(\xi) d \sigma_{\omega}(\xi), \quad \omega \in \Omega
$$

where $G$ is the Green function for $\Omega$ and $d \sigma_{\omega}$ is the harmonic measure relatively $\Omega$ and $\omega$.

Stokes theorem gives a similar formula for plurisubharmonic functions (cf. Demailly [4], [5] and Kołodziej [10]). Suppose $V, \varphi$ and $\psi \in P S H(\Omega) \cap L^{\infty}(\Omega)$ and define

$$
s(r)=\{z \in \Omega ; \varphi(z)=r\} ; \quad B(r)=\{z ; \varphi(z)<r\} .
$$

We assume that $B(r) \subset \subset \Omega \quad \forall r<0$. Consider

$$
\begin{aligned}
& \int_{S(r)} V d^{c} \varphi \wedge\left(d d^{c} \psi\right)^{k-1}=\text { (Stokes) } \\
& =\int_{B(r)} d V \wedge d^{c} \varphi \wedge\left(d d^{c} \psi\right)^{n-1}+\int V d d^{c} \varphi \wedge\left(d d^{c} \psi\right)^{n-1} \\
& =\int_{B(r)} d(\varphi-r) \wedge d^{c} V \wedge\left(d d^{c} \psi\right)^{n-1}+\int_{B(r)} V\left(d d^{c} \varphi\right) \wedge\left(d d^{c} \psi^{n-1}=\right.\text { Stokes) } \\
& =-\int_{B(r)}(\varphi-r) d d^{c} V \wedge\left(d d^{c} \psi\right)^{n-1}+\int_{B(r)} V d d^{c} \varphi \wedge\left(d d^{c} \psi\right)^{k-1} .
\end{aligned}
$$

Hence

$$
\begin{aligned}
\int_{B(r)} V\left(d d^{c} \varphi\right) \wedge\left(d d^{c} \psi\right)^{n-1}= & \int_{B(r)}(\varphi-r) d d^{c} V \wedge\left(d d^{c} \psi\right)^{n-1} \\
& +\int_{S(r)} V d^{c} \varphi \wedge\left(d d^{c} \psi\right)^{n-1} .
\end{aligned}
$$

We now claim that $d^{c} \varphi \wedge\left(d d^{c} \psi\right)^{n-1}$ is a positive measure on $S(r)$.

For let $0 \leq h \in C^{\infty}$ be given. Let $\epsilon>0$ and define $\varphi_{\epsilon}=\max \{\varphi, r-\epsilon\}$. Then

$$
\begin{aligned}
\int_{S(r)} h d^{c} \varphi & \wedge\left(d d^{c} \psi\right)^{n-1}=\int_{S(r)} h\left(d^{c} \varphi_{\epsilon}\right) \wedge\left(d d^{c} \psi\right)^{n-1} \\
& =\int_{S(r)} d \varphi_{\epsilon} \wedge d^{c} h \wedge\left(d d^{c} \psi\right)^{n-1}+\int_{B(r)} h\left(d d^{c} \varphi_{\epsilon}\right) \wedge\left(d d^{c} \psi\right)^{n-1} \\
& =\int_{r-\epsilon \leq \varphi<r} d \varphi_{\epsilon} \wedge d^{c} h \wedge\left(d d^{c} \psi\right)^{n-1}+\int_{r-\epsilon \leq \varphi<r} h d d^{c} \varphi_{\epsilon} \wedge\left(d d^{c} \psi\right)^{n-1}
\end{aligned}
$$




$$
\begin{aligned}
& =\int_{r-\epsilon \leq \varphi<r} d\left(\varphi_{\epsilon}-(r-\epsilon)\right) \wedge d^{c} h \wedge\left(d d^{c} \psi\right)^{n-1}+\int_{r-\epsilon \leq \varphi<r} h d d^{c} \varphi_{\epsilon} \wedge\left(d d^{c} \psi\right)^{n-1} \\
& =\int_{S(r)}\left(\varphi_{\epsilon}-(r-\epsilon)\right) d^{c} h \wedge\left(d d^{c} \psi\right)^{n-1}-\int_{r-\epsilon \leq \varphi<r}\left(\varphi_{\epsilon}-(r-\epsilon)\right) d d^{c} h \wedge\left(d d^{c} \psi\right)^{n-1} \\
& \quad+\int_{r-\epsilon \leq \varphi<r} h d d^{c} \varphi_{\epsilon} \wedge\left(d d^{c} \psi\right)^{n-1} .
\end{aligned}
$$

Here, the last term is nonnegative so

$$
\begin{aligned}
& \int_{S(r)} h d^{c} \varphi \wedge\left(d d^{c} \psi\right)^{n-1} \\
& \geq-\epsilon\left|\int_{B(r)} d d^{c} h \wedge\left(d d^{c} \psi\right)^{n-1}\right|-\epsilon \int\left|d d^{c} h \wedge\left(d d^{c} \psi\right)^{n-1}\right| \rightarrow 0, \quad \epsilon \rightarrow 0
\end{aligned}
$$

which proves the claim.

Example. Let $0 \leq V \in P S H(\Omega), \psi \in P S H \cap L^{\infty}(\Omega)$ and

$$
\varphi_{t}=\max [W(z, \xi),-t] .
$$

Then (1) gives

$$
\begin{aligned}
\int_{B(r)} V\left(d d^{c} \varphi_{t}\right) \wedge\left(d d^{c} \psi\right)^{n-1}= & \int_{B(r)}\left(\varphi_{t}-r\right) d d^{c} V \wedge\left(d d^{c} \psi\right)^{n-1} \\
& +\int_{s(r)} V d^{c} \varphi_{t} \wedge\left(d d^{c} \psi\right)^{n-1} .
\end{aligned}
$$

Letting $r \rightarrow 0$ we get

$$
\begin{aligned}
\int_{\Omega}-\varphi_{t} d d^{c} V \wedge\left(d d^{c} \psi\right)^{n-1} & \leq \int_{\Omega} V d d^{c} \varphi_{t} \wedge\left(d d^{c} \psi\right)^{n-1} \\
& \leq \sup _{\Omega} V \int_{\Omega} d d^{c} \varphi_{t} \wedge\left(d d^{c} \psi\right)^{n-1}
\end{aligned}
$$

so if we choose $\psi=\sum_{j=1}^{n}\left|z_{i}\right|^{2}$ then

(i)

$$
\int_{\Omega}-W(z, \xi) \Delta V \leq\|V\|_{L^{\infty}} \int_{\Omega} \Delta_{z} W(z, \xi),
$$

if we choose $\psi=V$, then

(ii)

$$
\int_{\Omega}-W(z, \xi)\left(d d^{c} V(z)\right)^{n} \leq\|V\|_{L \infty} \int_{\Omega} d d_{z}^{c} W(z, \xi)\left(d d^{c} V\right)^{n-1},
$$

and finally if $\psi=\varphi_{t}$,

$$
\int-\varphi_{t} d d^{c} V \wedge\left(d d^{c} \varphi_{t}\right)^{n-1} \leq \int V\left(d d^{c} \varphi_{t}\right)^{n}
$$


and so

$$
\int-W(z, \xi) d d^{c} V \wedge\left(d d^{c} W\right)^{n-1} \leq \int V\left(d d_{\xi}^{c} W(z, \xi)\right)^{n}
$$

4. Integrability of plurisubharmonic functions. Suppose $\mu$ is a positive measure on $\Omega$. How do we know there is a $\varphi \in P S H \cap L_{\operatorname{loc}}^{\infty}(\Omega)$ with $\left(d d^{c} \varphi\right)^{n}=\mu$ ? Here is a necessary condition.

Proposition 1. Let $R>1$ fixed, $B$ the unit ball. Then there exists a constant c such that

$$
\int_{\bar{B}}-\varphi\left(d d^{c} u\right)^{n} \leq c \int_{B}-\varphi d V
$$

for all $0 \geq \varphi \in P S H(R B)$ and $-1 \leq u \leq 0, u \in P S H(R B)$.

Proof. See [3, Prop. VI:2].

Let now $\Omega$ be hyperconvex with exhaustion function $\psi$. Let $\mu$ be a positive measure and assume $0 \geq V \in P S H \cap L^{\infty}(\Omega),\left(d d^{c} V\right)^{n}=\mu$. For $m>0$, define $V_{m}=\max (V, m \psi)$. Then, by $(1)$

so

$$
\begin{aligned}
\int V_{m}\left(d d^{c} \psi\right)^{n} & =\int \psi d d^{c} V_{m} \wedge\left(d d^{c} \psi\right)^{n-1} \\
& \leq \frac{1}{m} \int V_{m} d d^{c} V_{m} \wedge\left(d d^{c} \psi\right)^{n-1} \leq \ldots \leq \frac{1}{m^{n-1}} \int \psi\left(d d^{c} V_{m}\right)^{n} .
\end{aligned}
$$

$0 \leq \int-\psi\left(d d^{c} V_{m}\right)^{n} \leq m^{n-1} \int-V_{m}\left(d d^{c} \psi\right)^{n} \leq m^{n-1}\left(\sup _{z \in \Omega}-V(z)\right) \int\left(d d^{c} \psi\right)^{n}$. If $\tau(z) \equiv(2 \pi)^{n}$, we take $\psi(\xi)=W(z, \xi)$ and get

$$
(2 \pi)^{n} m^{n-1} V_{m}(z) \leq \int W(z, \xi)\left(d d^{c} V_{m} \xi\right)^{n} \leq 0 .
$$

If $\operatorname{supp} \mu$ is compact, then $V_{m}=V$ near the support of $\mu$ for $m$ large enough and therefore

$\left.0 \leq \int-W(z, \xi) d \mu(\xi) \leq m^{n-1} \int-V(\xi)\left(d d^{c} W(z, \xi)\right)^{n} \leq m^{n-1}\left(\sup _{\xi \in \Omega}-W\right) \tau(z)\right)$.

We are thus led to consider the pluricomplex potential $\Omega \ni z \mapsto \int W(z, \xi) d \mu(\xi)$ for positive measures $\mu$. We have just proved

TheOREM 2. Suppose $-1 \leq u \leq 0, u \in P S H(\Omega)$ and that $\Omega$ is strongly hyperconvex. Then

$$
\begin{aligned}
0 & \leq-\int W_{\Omega}(z, \xi)\left(d d^{c} \max \left[u(\xi), m W_{\Omega}(\eta, \xi)\right]\right)^{n} \\
& \leq-m^{n-1} \int \max \left[u(\xi), m W_{\Omega}(\eta, \xi)\right]\left(d d_{\xi}^{c} W_{\Omega}(z, \xi)\right)^{n} \leq m^{n-1} \tau(z), \quad z \in \Omega, \eta \in \Omega .
\end{aligned}
$$

5. A metric defined by $W$. It is known that $g_{\Omega}$ gives rise to an infinitesimal Finsler pseudometric, cf. [1], [8] and [9]. We show here that $W_{\Omega}$ also defines an infinitesimal Finsler pseudometric. 
Definition. Let $w \in \Omega, \xi \in \mathbb{C}^{n}$. We define

$$
T(\omega, \xi)=\varlimsup_{\substack{|l| \rightarrow 0 \\ l \in \mathbb{C}}} W_{\Omega}(w+l \xi, \omega)-\log |l| .
$$

Proposition 2. $T(\omega, \xi)$ is upper semicontinuous on $\Omega \times \mathbb{C}^{n}$.

Pro of. Note that $(\omega, \xi, l) \mapsto W_{\Omega}(\omega+l \xi, \omega)-\log |l|, l \neq 0$ is upper semicontinuous and subharmonic in $l$, for $\omega, \omega+l \xi \in \Omega$. Also, for $\omega, \xi$ fixed $W_{\Omega}(\omega+l \xi, \omega) \leq$ $c+\log |l|$. Therefore $W_{\Omega}(\omega+l \xi, \omega)-\log |l|$ has a uniquely determined subharmonic extension over $l=0$. Also

$$
T(\omega, \xi)=\varlimsup_{|l| \rightarrow 0} W_{\Omega}(\omega+l \xi, \omega)-\log |l|=\varlimsup_{r \searrow 0} W_{\Omega}(\omega+r \xi, \omega)-\log r .
$$

By the mean value property for subharmonic functions,

$$
\frac{1}{2 \pi} \int_{0}^{2 \pi}\left[W_{\Omega}\left(\omega+r e^{i \theta} \xi, \omega\right)-\log r\right] d \theta \searrow T(\omega, \xi), \quad r \searrow 0 .
$$

Note that for $r>0$ fixed the left hand side is upper semicontinuous in $(\omega, \xi)$ and since it decreases in $r$, the proposition follows.

Furthermore, $\exp T(\omega, t \xi)=|t| \exp T(\omega, \xi), t \in \mathbb{C}$ so $\exp T(\omega, \xi)$ defines an infinitesimal Finsler pseudometric.

\section{References}

[1] K. Azukawa, The invariant pseudometric related to negative plurisubharmonic function, Kodai Math. J. 10 (1987), 83-92.

[2] E. Bedford and J. P. Demailly, Two counterexamples concerning the pluri-complex Green function in $\mathbb{C}^{n}$. Indiana Univ. Math. J. 37 (1988), 865-867.

[3] U. Cegrell, Capacities in Complex Analysis. Aspects of Mathematics, 14, Vieweg, 1988

[4] J. P. Demailly, Mesures de Monge-Ampère et mesures pluri-sousharmoniques, Math. Z. 194 (1987), 519-564.

[5] —, Mesures de Monge-Ampère et caractérisation géométrique des variétés algébriques affines, Mém. Soc. Math. France 19 (1985), 1-125.

[6] M. Jarnicki and P. Pflug, Invariant Distances and Metrics in Complex Analysis, Walter de Gruyter \& Co., 1993.

[7] M. Klimek, Extremal plurisubharmonic functions and invariant pseudodistances, Bull. Soc. Math. France 113 (1985), 231-240.

[8] —, Infinitesimal pseudometrics and the Schwarz lemma, Proc. Amer. Math. Soc. 105 (1989), 134-140.

[9] - Pluripotential Theory, Oxford Science Publications, 1991.

[10] S. Kołodziej, The logarithmic capacity in $\mathbb{C}^{n}$, Ann. Polon. Math. 48 (1988), 253-267. 